\title{
Preditores Individuais e Situacionais de Auto e Heteroavaliação de Impacto do Treinamento no Trabalho
}

\author{
Pedro Paulo Murce Meneses \\ Gardênia Abbad
}

\begin{abstract}
Resumo
Este estudo analisou o relacionamento entre características de treinamentos e suas clientelas, suporte à transferência e impacto do treinamento no trabalho. As amostras foram obtidas no Distrito Federal, junto a três organizações de médio e grande porte. A coleta de dados ocorria no primeiro dia de curso, quando eram aplicadas medidas de auto-eficácia, locus de controle e motivação para o treinamento, e entre um e três meses finalizados os cursos, momento em que eram aplicadas medidas de impacto do treinamento e de suporte à transferência. Estes instrumentos apresentaram índices psicométricos satisfatórios. Sobre a análise do relacionamento entre as variáveis mencionadas, somente suporte psicossocial percebido pelos participantes e auto-eficácia, e suporte psicossocial percebido pelas chefias e colegas de trabalho e quantidade de instrutores por turma, contribuíram, respectivamente, na explicação da variabilidade de auto e heteroavaliação de impacto do treinamento. Quando a variável critério foi heteroavaliação do impacto, nenhuma variável auto-referente contribuiu para a sua explicação. Talvez isso indique que a participação das variáveis na explicação do impacto se deva tão somente a natureza das medidas utilizadas. Sugere-se a diversificação da natureza de medidas utilizadas.
\end{abstract}

Palavras-chaves: avaliação de treinamento; auto-eficácia; locus de controle; suporte à transferência; impacto do treinamento no trabalho.

\begin{abstract}
This research sought to analyze the relationship among training characteristics, trainee attributes, support to transfer, and training impact, was analyzed. The samples were gathered at Distrito Federal, Brazil, on three organizations, of medium and large size. The data were collected in two moments. On the first day of training, self-efficacy, locus of control, and training motivation measures were used. About one to three months after the training end, measures of training impact, and support to transfer were used. All the measures obtained satisfactory psychometrics results. Referring to the results of the relationship analyses, just psycho-social support to transfer perceived by trainees, and self-efficacy, and also psycho-social support perceived by supervisors and work peers, and quantity of trainers for each class, explained training impact perceived by trainees, and by supervisors and peers, respectively. When the dependent variable was training impact evaluation by supervisors and peers, none of the self-referents variables explained it. Maybe, the contribution of the previous variables to the impact occurred just because of the used measures' nature. In the future, studies should use measures of different natures in order to check if the independent variables' contribution to training impact keeps stable.
\end{abstract}

Key words: training assessment; self-efficacy; locus of control; support to transfer; training work impact. 


\section{INTRODUÇÃO}

O mundo do trabalho é marcado por acirrada competição entre empresas, por nichos de mercados cada vez menores e profissionais, exigência de novas habilidades cognitivas e afetivas, entrada de minorias nas organizações e constante inovação tecnológica, responsáveis pela rápida obsolescência da mão-de-obra qualificada. Nesse cenário, programas de treinamento e desenvolvimento (T\&D), eficazes instrumentos de aprendizagem das novas habilidades exigidas, tornamse essenciais. Esta relevância conferida à área de T\&D é convertida em elevados investimentos financeiros, desperdiçados freqüentemente pela inexistência de estudos criteriosos e sistemáticos sobre processos de transferência de aprendizagem, mudança de comportamento ou impacto de treinamentos no trabalho.

A produção de conhecimentos e tecnologias sobre sistemas de T\&D tornase, portanto, fundamental. Entretanto pouquíssimas organizações investem em sistemas adequadamente estruturados e integrados. A maioria dos programas, por exemplo, é avaliada ainda somente no nível de satisfação de seus participantes com o conteúdo ministrado e o desempenho do instrutor. No meio acadêmico, apesar das muitas investigações sobre o tema, nota-se escassez de estudos sobre o relacionamento das variáveis pessoais com eficácia de programas de T\&D.

Para Tannenbaum et al. (1991) a eficácia destes programas depende das características dos treinandos. Historicamente, pesquisas sobre o tema têm-se preocupado em selecionar participantes mais propensos a ser bem sucedidos em programas de treinamento. Trata-se da noção da treinabilidade, na qual características individuais como, por exemplo, motivação para o treinamento, auto-eficácia, locus de controle, determinam quem se beneficia, em maior ou menor proporção, de programas de $T \& D$.

Este relato, analisando as relações das variáveis de características de treinamentos e suas clientelas e de suporte à transferência com o nível de avaliação de impacto do treinamento no trabalho, pretende contrapor-se à noção da treinabilidade. Seus resultados não visam à seleção de indivíduos treináveis, mas à adequação dos programas de $T \& D$ a determinados perfis de clientela de participantes. A seguir, é apresentada ao leitor a área de $\mathrm{T} \& \mathrm{D}$, seus subsistemas e alguns modelos de avaliação. 


\section{Treinamento e Desenvolvimento}

Diferentemente de desenvolvimento e educação, treinamento se refere a intervenções sistematicamente planejadas para promoverem melhorias de desempenho em tarefas atuais. Para Bastos (1991) uma das maneiras de diferenciar treinamento dos demais é pelo critério da intencionalidade em produzir melhorias do desempenho e do controle exercido pela organização sobre o processo de treinamento.

Em face da inconstância do cenário mundial do trabalho, atividades designadas para cargos, funções ou ocupações, acompanhando as novas tendências, tornam-se cada vez mais complexas e exigentes. Organizações, pressionadas pela insegurança gerada pela instalação deste novo cenário, passam a entender treinamento como intervenção contínua, voltada ao preparo de indivíduos para um futuro profissional imprevisível, que se confunde, novamente, com as noções de educação e desenvolvimento. A presente pesquisa buscou investigar principalmente a eficácia de treinamentos, relacionando-a a características de treinamentos, clientelas e ambientes organizacionais.

Para Borges-Andrade e Abbad (1996), entre os propósitos do treinamento, destacam-se a identificação e superação de deficiências nos desempenhos, a preparação de empregados para novas funções e o retreinamento de adaptação de mão-de-obra na introdução de novas tecnologias. Treinamento é entendido como desenvolvimento sistemático de padrões comportamentais de conhecimentos, habilidades e atitudes, necessários ao desempenho adequado de uma tarefa ou trabalho (Latham, 1988).

Goldstein e Gilliam (1990) definem treinamento como um modelo de tecnologia instrucional composto por avaliação de necessidades, experiências de aprendizagem precisamente controladas e planejadas para alcançar objetivos instrucionais, uso de critérios de desempenhos e coleta de informações para fornecer feedback no concernente aos efeitos do sistema, etapa sobre a qual recai o interesse do presente relato de pesquisa

Assim a etapa de avaliação de treinamento compreende a coleta sistemática de informações descritivas e de julgamentos, a fim de tornar efetiva qualquer decisão relacionada à seleção, adoção, valoração e modificação das várias atividades instrucionais. Entre suas razões, destaca-se a necessidade de torná-los externamente válidos ou potencialmente capazes de provocarem modificações em ambientes organizacionais. É a melhor oportunidade para discutir os treina- 
mentos, seus eventuais papéis na mudança organizacional e social e, perante a prática vigente de $\mathrm{T} \& \mathrm{D}$, argumentar a favor do desenvolvimento de sistemas de treinamento completos e bem estruturados.

A sua execução poderia centrar-se em diversos níveis, sendo os mais difundidos os de reações, aprendizagem e comportamento no cargo (Hamblin, 1978). Propostos há mais de 30 anos, tais níveis não exploram inúmeras outras variáveis, também capazes de influenciar a eficácia de treinamentos. Nesse sentido, diversos modelos de avaliação de treinamento foram desenvolvidos. Entre eles, destacase o Modelo de Avaliação Integrado Somativo (MAIS), proposto por BorgesAndrade (1982), que, além de contemplar variáveis internas a programas de treinamentos, prevê a influência de variáveis externas, como o ambiente, sobre os resultados dos treinamentos.

Após o desenvolvimento deste modelo, outros surgiram na tentativa de elevar o poder explicativo de algumas variáveis sobre os efeitos de treinamentos. Abbad (1999) desenvolveu um modelo integrado de avaliação do impacto do treinamento no trabalho (IMPACTO), cuja avaliação enfoca variáveis de suporte organizacional, características do treinamento e clientelas, bem como os níveis de avaliação de reação, aprendizagem e impacto do treinamento no trabalho. A partir deste modelo, propõe-se outro mais parcimonioso (vide Figura 1), que possibilita a investigação do relacionamento de características do treinamento, suas clientelas e de suporte à transferência com o nível de avaliação de impacto do treinamento no trabalho.

Figura 1: Modelo de Meneses

1. Características dos Treinamentos

2. Características da Clientela

2.1 Auto-eficácia

2.2 Motivação para o Treinamento

2.3 Locus de Controle

2.4 Características Demográficas

3. Suporte à Transferência

Fonte: Meneses (2002).

Explicitemos cada um dos componentes da figura.

- Características dos Treinamentos. Natureza predominante do objetivo principal do curso (cognitivo, afetivo ou psicomotor) e quantidade de instrutores por treinamento analisado. 
. Características da Clientela. Auto-eficácia: auto-avaliações dos indivíduos acerca de suas capacidades para obter sucesso em suas realizações. Motivação para o treinamento: grau de esforço despendido para assimilar os conteúdos do treinamento e transferi-los para o trabalho, bem como valor atribuído ao treinamento como estratégia de resolução de problemas de desempenho no trabalho. Locus de controle: crenças dos indivíduos sobre as fontes que controlam seu próprio comportamento e outros eventos. Características demográficas: gênero, faixa etária, nível de escolaridade, estado civil, ter filhos ou não, tipo de religião ou crença e organização de origem.

. Suporte à Transferência do Treinamento. Opinião dos participantes, chefias e colegas de trabalho a respeito do nível de apoio psicossocial e material fornecido pelo ambiente ao uso eficaz das novas habilidades no trabalho.

Impacto em Amplitude do Treinamento no Trabalho. Auto e heteroavaliações por parte da chefia e colegas de trabalho, quanto aos efeitos produzidos pelo treinamento nos níveis de desempenho, motivação, autoconfiança e abertura a mudanças nos processos de trabalhos, em que estão envolvidos os indivíduos treinados.

Na seção seguinte, apresentam-se breves sínteses da literatura acerca de cada um dos componentes descritos.

\section{ReVisão de Literatura}

Em relação a características dos programas de $T \& D$, conforme revisão de literatura realizada por Borges-Andrade e Abbad (1996), praticamente não há produção tecnológica ou de conhecimento, ou pelo menos dela não houve divulgação, referente aos passos para a elaboração de projetos na área e seus efeitos sobre reações, aprendizagem e impacto do treinamento no trabalho. Provavelmente, o desinteresse pelo tema decorre da excessiva ênfase dada pelos profissionais a questões concernentes a projetos de treinamento nas décadas de 60 e 70 do século anterior, ou ainda, do predomínio atual de questões moleculares acerca de interações de processos cognitivos básicos, métodos e estratégias instrucionais (Abbad, 1999).

Sobre motivação para o treinamento, os modelos e preditores têm variado amplamente nas duas últimas décadas e o resultado tem sido uma rede extensa $\mathrm{e}$ útil de trabalho referente à motivação para treinamento, ao custo, porém, da falta de convergência nos resultados e de clareza conceitual. Para Salas e CannonBowers (2001), apesar de a literatura ser clara a respeito da influência da 
motivação para o treinamento sobre resultados de aprendizagem, as imprecisões conceituais ainda persistem.

Colquitt, LePine e Noe (2000) buscaram integrar, por meio de metanálise, a teoria existente acerca da motivação para o treinamento, a partir de extensa revisão de resultados de pesquisa, na qual variáveis como locus de controle, auto-eficácia, características demográficas e clima organizacional para transferência estariam relacionadas com aprendizagem e transferência do treinamento. Os resultados da pesquisa conduzida pelos autores indicaram que locus de controle e motivação para o treinamento se relacionaram fortemente $(\mathrm{r}=0,46)$, sugerindo que indivíduos com locus de controle interno tendem a apresentar índices mais elevados de motivação para o treinamento. Auto-eficácia também esteve moderadamente relacionada à motivação para o treinamento $(\mathrm{r}=$ 0,42), indicando que indivíduos com escores elevados de auto-eficácia apresentam também índices maiores de motivação para o treinamento. Quanto às variáveis situacionais, o clima favorável de trabalho relacionou-se positivamente com a motivação para o treinamento $(r=0,39)$.

Quanto à auto-eficácia, conforme revisão de literatura realizada por Salas e Cannon-Bowers (2001), o conceito, largamente estudado na última década, assume grande importância em treinamento, à medida que propicia melhores resultados de aprendizagem e desempenho pós-treinamento. De acordo com a revisão realizada por Borges-Andrade (1997), inúmeras pesquisas apontam a autoeficácia como importante variável explicativa do sucesso dos participantes em treinamentos de diferentes domínios e atividades.

Colquitt, LePine e Noe (2000) ressaltam que a auto-eficácia se tem mostrado positiva e fortemente relacionada ao desempenho no trabalho. Em situações de treinamento, o relacionamento entre auto-eficácia e escolha, esforço e persistência na realização da tarefa torna mais provável o estabelecimento de uma relação positiva entre auto-eficácia e resultados de treinamento. No estudo conduzido pelos autores, auto-eficácia relacionou-se, entre outros, com motivação para aprendizagem $(r=0,42)$, transferência do treinamento $(r=0,47)$, conhecimento declarativo $(\mathrm{r}=0,30)$, aquisição de habilidades $(\mathrm{r}=0,32)$, desempenho no trabalho $(r=0,22)$ e reações $(r=0,17)$.

Outra variável de interesse, que também parece afetar resultados imediatos de treinamentos, é locus de controle, característica que vem sendo pouco estudada, provavelmente porque outras variáveis como motivação e auto-eficácia têm sido consideradas melhores preditoras de resultados de treinamento.

Apesar da pouca atenção, percebe-se a relativa importância de locus de controle em situação de treinamento, como sugerido, por exemplo, por Colquit, LePine e 
Noe (2000). Em uma revisão de literatura, os autores demonstram a forte relação entre locus de controle e motivação para aprender $(\mathrm{r}=-0,46)$, indicando que pessoas com locus de controle interno tendem a apresentar alto nível de motivação. Locus de controle também esteve moderadamente relacionado a conhecimento declarativo $(\mathrm{r}=0,21)$ e transferência $(\mathrm{r}=0,27)$, com efeitos opostos. Pessoas com locus de controle externo aprenderam mais e tiveram níveis mais altos de transferência do que indivíduos com locus de controle interno.

No concernente a características demográficas, apesar de estas variáveis serem mencionadas em estudos sobre treinamento, na maioria das vezes são utilizadas unicamente como fontes de controle estatístico. Há pouquíssimos estudos em que características demográficas assumem papel central e quase não há pesquisa relacionando tais variáveis com efeitos de treinamentos. Entre as mais estudadas, destacam-se o gênero, com resultados ainda contraditórios, e a idade, cujos relatos científicos evidenciam uma relação negativa entre esta característica e aprendizagem (Abbad, 1999; Colquitt, LePine e Noe, 2000).

Em relação às variáveis situacionais, segundo Tannenbaum e Yukl (1992) a aprendizagem é necessária, mas não essencial para uma mudança de comportamento no trabalho. Ambientes pós-treinamento desempenhariam também papel importante na determinação desta mudança. Por exemplo, limitações no ambiente pós-treinamento poderiam prejudicar a aplicação, no trabalho, das habilidades adquiridas por meio de treinamento.

Para Abbad (1999) clima ou suporte à transferência de treinamento é, sem dúvida, um componente crítico no estudo das variáveis que afetam a eficácia de programas instrucionais. Como descrito por Sallorenzo (2001), os resultados de pesquisas nacionais, como as realizadas por Paula (1992), Leitão (1994), Abbad (1999) e Brito (1999), apontam a presença de relacionamentos consistentes entre suporte à transferência e impacto do treinamento no trabalho.

Quanto ao impacto do treinamento no trabalho, entende-se o efeito indireto do treinamento no desempenho dos treinandos, na motivação e/ou atitudes, como sugerido por Abbad, Borges-Andrade e Sallorenzo (19-). Esses autores propõem uma análise do conceito de impacto, já que ele abrangeria também os conceitos de transferência de treinamento e desempenho no trabalho. Transferência estaria relacionada à aplicação correta dos conhecimentos, habilidades e atitudes adquiridos em treinamentos para situações de trabalho; entretanto o impacto não deve ser reduzido somente à transferência positiva de treinamento.

Conforme Abbad (1999), habilidades aprendidas tais como as metacognitivas, as estratégias de autogerenciamento e o uso de ferramentas computacionais, entre outras, podem afetar o desempenho individual em diversas atividades rea- 
lizadas dentro da organização. Habilidades não podem ser carregadas, como sugerido metaforicamente pela expressão transferência de treinamento. Elas são disposições. O foco do treinamento é em desempenhos, meios de execução de tarefas alinhados a certos critérios e situações.

\section{FOCO DO EsTUdO}

Atualmente, uma das questões empíricas de maior interesse na área de avaliação de treinamento diz respeito aos efeitos de eventos instrucionais, características da clientela e do ambiente organizacional sobre os níveis de desempenho no trabalho. Como sugerido por Sallorenzo (2001), alternativas de projetos de pesquisas devem investir no estudo de características individuais como determinantes de incremento de impacto de treinamento no trabalho, como, por exemplo, locus de controle e auto-eficácia. Da mesma forma, Borges-Andrade e Abbad (1996) propõem uma agenda na qual características da clientela devem ser foco de alguma atenção no Brasil, tanto em termos de desenvolvimento de medidas de avaliação, quanto em termos de seus efeitos na organização e no treinamento. Um leitor apressado pode questionar se estes estudos não instigam a seleção de indivíduos capazes de ser mais bem treinados.

Trata-se da controversa noção de treinabilidade, que pode levar à idéia errônea da distinção de dois tipos de indivíduos em uma organização, os treináveis e os não treináveis. Entretanto esse trabalho fundamenta-se na premissa de que qualquer indivíduo é capaz de aprender e transferir suas novas habilidades para situações de trabalho, desde que o desenho instrucional e as condições de apoio à aprendizagem e à transferência o estimulem a engajar-se nesse processo de mudança comportamental.

Na prática, pesquisas sobre o tema são úteis à medida que permitem, já na etapa de avaliação de necessidades, identificar que tipos de treinamentos devem ser desenvolvidos a fim de maximizar, para cada indivíduo, seus efeitos. Desta forma, o principal interesse da presente pesquisa recai sobre a investigação das contribuições de variáveis individuais, do próprio treinamento e de suporte à transferência na explicação da variabilidade de impacto do treinamento no trabalho.

\section{Método}

Nesta seção são apresentadas as principais características dos treinamentos e da amostra de participantes analisada. Também são descritos os instrumentos de 
medida utilizados e, por fim, são enunciados os procedimentos de coleta de dados empregados e as análises estatísticas realizadas.

\section{Treinamentos e Participantes}

Constituíram a amostra treinandos que responderam a todos os instrumentos utilizados e, ainda, que tiveram os instrumentos de suporte à transferência e impacto do treinamento avaliados por suas chefias e colegas de trabalho. Por tal motivo de emparelhamento de respostas, a amostra foi formada por 366 casos de participações em 23 treinamentos distintos ofertados por três organizações, públicas e privadas, sedeadas no Distrito Federal. Destes, 20 eram de natureza predominantemente cognitiva, um de natureza afetiva e dois de natureza psicomotora. Como alguns treinamentos ocorreram mais de uma vez, 53 turmas, com média de sete alunos, foram constituídas. A maioria deles (34) foi ministrada por um instrutor. A Tabela 1 apresenta o número de participações em treinamentos nas organizações e o perfil demográfico da amostra de treinandos.

Tabela 1: Principais Características da Amostra de Treinandos

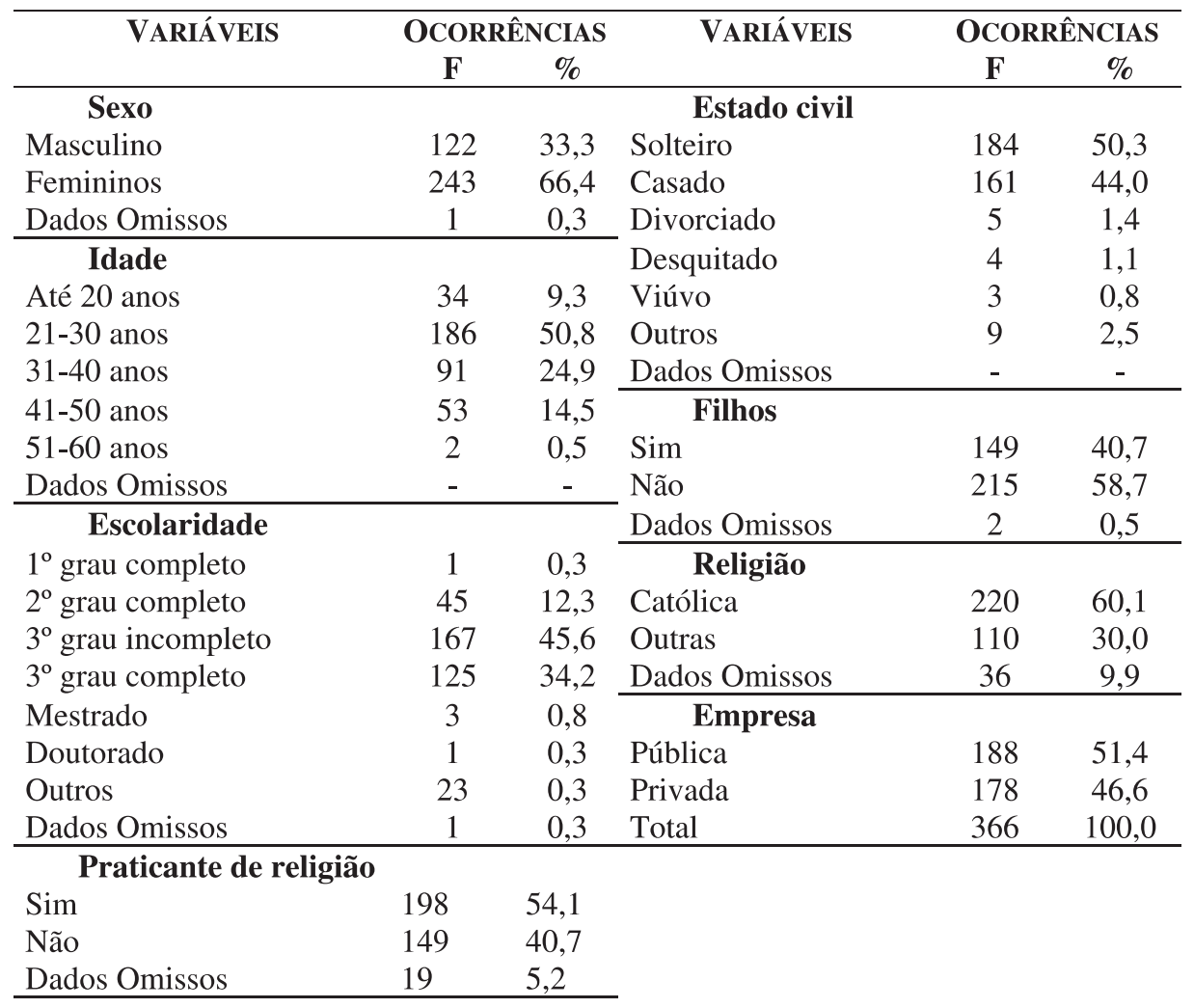




\section{Instrumentos de Medida}

Os seguintes instrumentos foram utilizados: Auto-eficácia, Locus de Controle, Motivação para o Treinamento, Impacto em Amplitude do Treinamento no Trabalho e Suporte à Transferência. Os dados demográficos foram coletados no questionário de Locus de Controle. Para as avaliações de impacto do treinamento no trabalho e suporte à transferência, também foram utilizadas medidas de heteroavaliação (chefias e colegas de trabalho). O Quadro 1 apresenta os questionários utilizados, relacionando-os com os componentes do modelo de avaliação proposto. Apesar de não ter sido considerado como variável de interesse na presente pesquisa, vale ressaltar que também foi utilizado, em duas das organizações estudadas, o instrumento de Reação, desenvolvido e validado estatisticamente por Abbad (1999).

\section{Quadro 1: Componentes do Modelo de Avaliação, Questionários e Itens Correspondentes}

\begin{tabular}{|c|c|c|}
\hline \multicolumn{3}{|c|}{ COMPONENTE 1 - CARACTERÍSTICAS DOS TREINAMENTOS } \\
\hline ROTEIRO & ITENS & $\begin{array}{l}\mathrm{N}^{\circ} . \mathrm{DE} \\
\text { ITENS }\end{array}$ \\
\hline Análise Documental & $\begin{array}{l}\text { Natureza do Objetivo Principal do } \\
\text { Curso e Quantidade de Instrutores } \\
\text { por Curso }\end{array}$ & - \\
\hline \multicolumn{3}{|c|}{ COMPONENTE 2 - CARACTERÍSTICAS DA CLIENTELA ${ }^{(1)}$} \\
\hline QUESTIONÁRIOS & ITENS & $\begin{array}{l}\mathrm{N}^{\mathrm{o}} . \mathrm{DE} \\
\text { ITENS }\end{array}$ \\
\hline \multirow[t]{2}{*}{ Auto-eficácia } & Auto-eficácia & 15 \\
\hline & Motivação para a Aprendizagem & 4 \\
\hline \multirow[t]{2}{*}{ Locus de Controle } & Locus de Controle & 12 \\
\hline & Informações Demográficas & 13 \\
\hline \multicolumn{3}{|c|}{ COMPONENTE 3 - SUPORTE À TRANSFERÊNCIA } \\
\hline QUESTIONÁRIOS & ITENS & $\begin{array}{l}\mathrm{N}^{\circ} . \mathrm{DE} \\
\text { ITENS }\end{array}$ \\
\hline \multirow{3}{*}{$\begin{array}{l}\text { Suporte à Transferência percebido pelos } \\
\text { participantes, chefias e colegas de trabalho }\end{array}$} & Fatores Situacionais de Apoio & 9 \\
\hline & Suporte Material & 6 \\
\hline & $\begin{array}{l}\text { Consequiências Associadas ao Uso } \\
\text { das Novas Habilidades }\end{array}$ & 7 \\
\hline \multicolumn{3}{|c|}{ COMPONENTE 4 - IMPACTO DO TREINAMENTO } \\
\hline QUESTIONÁRIOS & ITENS & $\mathrm{N}^{\mathrm{o}} . \mathrm{DE}$ \\
\hline $\begin{array}{l}\text { Auto e Heteroavaliação do Impacto do } \\
\text { Treinamento }\end{array}$ & $\begin{array}{l}\text { Impacto do Treinamento no } \\
\text { Trabalho }\end{array}$ & $\begin{array}{l}\text { ITENS } \\
12\end{array}$ \\
\hline
\end{tabular}

Todos os instrumentos utilizados nesta pesquisa foram submetidos a procedimentos de validação estatística (análise dos principais componentes e análises fatoriais), a fim de investigar sua adequação aos propósitos determinados. A Tabela 2 apresenta os principais resultados destes procedimentos. 


\section{Tabela 2: Principais Resultados da Validação Estatística dos Instrumentos Utilizados}

\begin{tabular}{|c|c|c|c|c|c|c|}
\hline Instrumentos & Escalas Obtidas & KMO & $\begin{array}{l}\mathrm{N}^{\circ} \mathrm{de} \\
\text { Itens }\end{array}$ & $\begin{array}{l}\text { Valores } \\
\text { Próprios } \\
\end{array}$ & $\begin{array}{c}\% \\
\text { Variância } \\
\end{array}$ & Alphas \\
\hline Motivação & $\begin{array}{l}\text { Motivação para o } \\
\text { Treinamento }\end{array}$ & 0,68 & 4 & 2,23 & 44,80 & 0,69 \\
\hline \multirow{2}{*}{ Auto-eficácia } & AE para itens favoráveis & & 9 & 3,24 & 18,60 & 0,78 \\
\hline & AE para itens desfavoráveis & 0,84 & 4 & 3,47 & 19,69 & 0,91 \\
\hline \multirow{2}{*}{$\begin{array}{l}\text { Locus de } \\
\text { Controle }^{(1)}\end{array}$} & Internalidade & 0,93 & 12 & 4,37 & 10,16 & 0,80 \\
\hline & Externalidade & & 24 & 8,73 & 22,66 & 0,91 \\
\hline \multirow{2}{*}{$\begin{array}{c}\text { Suporte Percebido } \\
\text { pelos } \\
\text { Participantes }\end{array}$} & $\begin{array}{l}\text { Suporte Psicossocial } \\
\text { percebido pelos participantes }\end{array}$ & 0,89 & 13 & 7,32 & 31,03 & 0,89 \\
\hline & $\begin{array}{l}\text { Suporte Material percebido } \\
\text { pelos participantes }\end{array}$ & & 6 & 2,71 & 10,11 & 0,78 \\
\hline \multirow{2}{*}{$\begin{array}{c}\text { Suporte Percebido } \\
\text { pelas Chefias e } \\
\text { Colegas de } \\
\text { Trabalho }\end{array}$} & $\begin{array}{l}\text { Suporte Psicossocial } \\
\text { percebido pelas Chefias e } \\
\text { Colegas }\end{array}$ & 0,91 & 13 & 6,65 & 27,86 & 0,87 \\
\hline & $\begin{array}{l}\text { Suporte Material percebido } \\
\text { pelas Chefias e Colegas }\end{array}$ & & 5 & 2,75 & 10,46 & 0,88 \\
\hline \multirow{2}{*}{$\begin{array}{l}\text { Auto-avaliação de } \\
\text { Impacto } \\
\text { Heteroavaliação } \\
\text { de Impacto }\end{array}$} & Auto-avaliação de Impacto & 0,92 & 12 & 5,99 & 45,56 & 0,87 \\
\hline & Heteroavaliação de Impacto & 0,93 & 12 & 6,18 & 49,94 & 0,88 \\
\hline
\end{tabular}

Vale ressaltar que os resultados apresentados na Tabela 2 permitiram que as variáveis, antecedentes e critério, fossem constituídas a partir das médias das respostas dos participantes aos itens que as compuseram. Exceto para auto e heteroavaliação de impacto, utilizadas como variáveis critério, as demais constituíram variáveis antecedentes dos modelos testados. É importante mencionar que as variáveis antecedentes suporte psicossocial e material à transferência percebido pelos participantes e pelas chefias e colegas de trabalho, foram somente consideradas nos modelos cujas variáveis critério eram, respectivamente, auto e heteroavaliação do impacto do treinamento no trabalho.

Também foram utilizadas como variáveis antecedentes, após as categorias de respostas terem sido dicotomizadas, em alguns casos, os seguintes dados demográficos coletados: gênero (masculino ou feminino), faixa etária (até 30 anos ou de 31 a 60 anos), nível de instrução (até $3^{\circ}$ grau incompleto ou de $3^{\circ}$ grau completo a doutorado), estado civil (solteiros ou outros), organização de origem, religião (católica ou outras) e filhos ( sim ou não). Foi também utilizada a quantidade de instrutores por turma de treinamento analisado. A variável natureza do treinamento não pôde ser utilizada, já que mais de $95 \%$ dos cursos eram de natureza predominantemente cognitiva. 


\section{Coleta e Análise de Dados}

A coleta de dados ocorreu em dois momentos distintos. No início dos treinamentos, os participantes avaliaram os instrumentos de auto-eficácia, locus de controle, motivação para o treinamento e forneceram algumas informações demográficas. Esses instrumentos foram aplicados, em sessão única de avaliação, em todos os participantes presentes no primeiro dia de curso. Embora desconsiderado nas análises posteriores, o instrumento de reação, como mencionado, foi aplicado no último dia de curso. Entre um e três meses após a conclusão dos treinamentos, os participantes, chefias e colegas de trabalho receberam, via correio eletrônico, os instrumentos de auto-avaliação de impacto e suporte à transferência percebido pelos participantes e heteroavaliação de impacto e suporte à transferência percebido pelas chefias e colegas de trabalho, respectivamente. No caso de uma das organizações estudadas, também foi utilizada avaliação em papel. A Figura 2 evidencia o esquema utilizado para coleta de dados.

\section{Figura 2: Esquema de Coleta de Dados e Instrumentos Utilizados em cada Etapa}

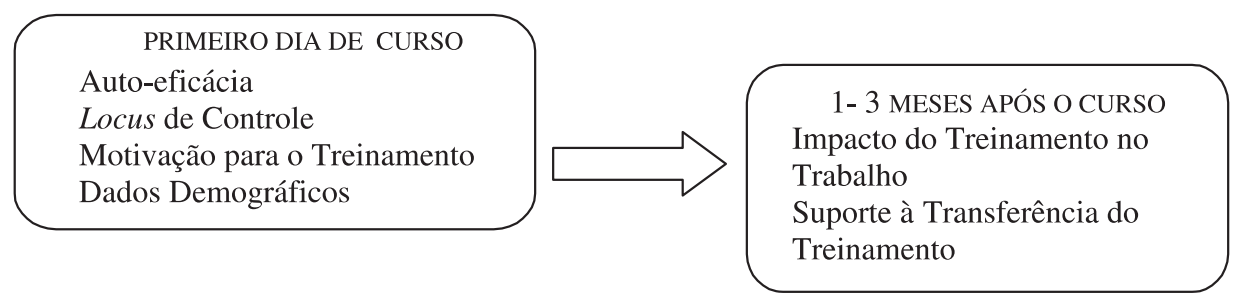

As informações coletadas nos dois momentos mencionados foram posteriormente inseridas em um arquivo de dados, gerado por meio do programa SPSS for Windows, Versão 10.0, para que pudessem ser analisadas por meio de análises de regressão múltipla padrão.

Anteriormente às análises mencionadas, porém, as médias das respostas dos 366 casos de participação nas variáveis antecedentes e critério foram examinadas por meio de vários programas do SPSS, quanto à ocorrência de valores ausentes e adequação das distribuições em relação aos pressupostos para análises multivariadas.

As variáveis mencionadas apresentaram valores omissos em menos de $5 \%$ dos casos e por isso foi utilizado tratamento pairwise para dados ausentes. Apesar de refletidas, devido a índices negativos de assimetria, e transformadas as distribuições das médias das respostas às escalas de motivação para o treinamento e suporte material à transferência percebido pelos participantes do treinamento 
(método raiz quadrada), devido aos índices elevados de assimetria e achatamento, os resultados não apresentaram melhorias significativas. Assim foi escolhido não transformar qualquer distribuição. Considerando a distância Mahalanobis com $\alpha=0,001$, dois outliers multivariados foram encontrados e excluídos do arquivo de dados. Esses exames indicaram a adequação dos dados obtidos aos pressupostos exigidos pela análise de regressão múltipla padrão, cujos resultados serão descritos a seguir.

\section{Resultados}

Em relação à variável critério auto-avaliação do impacto do treinamento no trabalho, os dados $(\mathrm{N}=364)$ foram submetidos à análise de regressão múltipla padrão. A Figura 3 apresenta os coeficientes de regressão padronizados $(\beta)$ e as contribuições individuais das variáveis estatisticamente significativas, bem como $\mathrm{o} \underline{\mathrm{R}}^{2} \mathrm{e} \mathrm{o} \underline{\mathrm{R}}^{2}$ ajustado. O coeficiente de regressão múltipla $\underline{\mathrm{R}}$ foi significativamente diferente de zero, $\underline{\mathrm{F}}(15,298)=20,86, \mathrm{p}<0,001$. Para os dois coeficientes de regressão que diferiram significativamente de zero, limites de confiança de $95 \%$ foram considerados. Os intervalos de confiança variaram de 0,12 a 0,43 para auto-eficácia e de 0,45 a 0,61 para suporte material à transferência percebido pelos participantes dos treinamentos.

Entre as variáveis antecedentes utilizadas, somente duas contribuíram significativamente para a explicação de auto-avaliação de impacto do treinamento no trabalho: suporte psicossocial percebido pelos participantes do treinamento $\left(\mathrm{sr}^{2}=0,27\right)$ e auto-eficácia $\left(\mathrm{sr}^{2}=0,02\right)$. As outras 13 variáveis antecedentes combinadas contribuíram em $20 \%$ para a variabilidade compartilhada. Agrupadas, as 15 variáveis antecedentes explicavam $51 \%$ (49\% ajustado) da variabilidade de impacto do treinamento no trabalho percebido pelos participantes.

\section{Figura 3: Modelo Empírico Resultante do Teste do Modelo Teórico para Auto-Avaliação do Impacto do Treinamento no Trabalho}

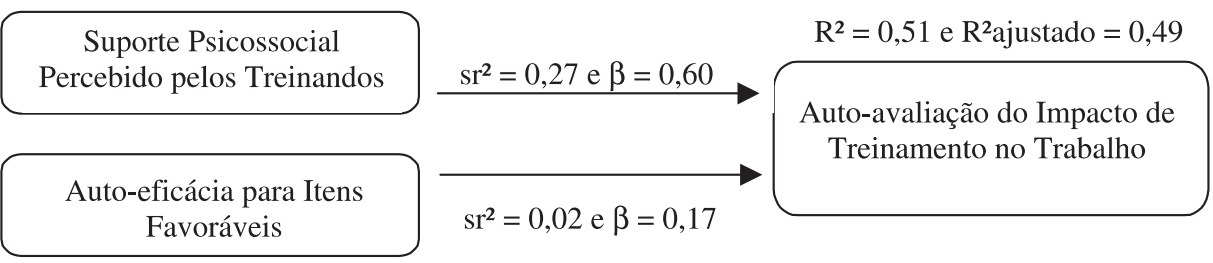


Quanto à variável critério heteroavaliação do impacto do treinamento no trabalho, os dados $(\mathrm{N}=364)$ também foram submetidos à análise de regressão múltipla padrão. A Figura 4 apresenta os coeficientes de regressão padronizados $(\beta)$ e as contribuições individuais das variáveis estatisticamente significativas, bem como $\mathrm{o} \underline{\mathrm{R}}^{2}$ e o $\underline{\mathrm{R}}^{2}$ ajustado. Pode ser observado que $\underline{\mathrm{R}}$ foi significativamente diferente de zero, $\underline{F}(15,298)=19,66, p<0,001$. Para os dois coeficientes de regressão que diferiram significativamente de zero, limites de confiança de $95 \%$ foram considerados. Os intervalos de confiança variaram de 0,50 a 0,70 para suporte psicossocial percebido pelas chefias dos participantes do treinamento e de $-0,35$ a $-0,14$ para quantidade de instrutores por turma de treinamento.

Entre todas as variáveis antecedentes consideradas, somente duas contribuíram significativamente para a explicação de heteroavaliação do impacto do treinamento no trabalho percebido pelos participantes: suporte psicossocial percebido pelas chefias dos participantes do treinamento $\left(\mathrm{sr}^{2}=0,22\right)$ e quantidade de instrutores por turma de treinamento $\left(\mathrm{sr}^{2}=0,03\right)$. O restante, 13 variáveis antecedentes, contribuiu em $22 \%$ para a variabilidade compartilhada. Agrupadas, todas as 15 variáveis antecedentes explicaram 50\% (47\% ajustado) da variabilidade de impacto do treinamento no trabalho percebido pelas chefias dos participantes. Em seguida, os resultados aqui apresentados são discutidos a partir da revisão de literatura descrita.

\section{Figura 4: Modelo Empírico Resultante do Teste do Modelo Teórico para Heteroavaliação do Impacto do Treinamento no Trabalho}

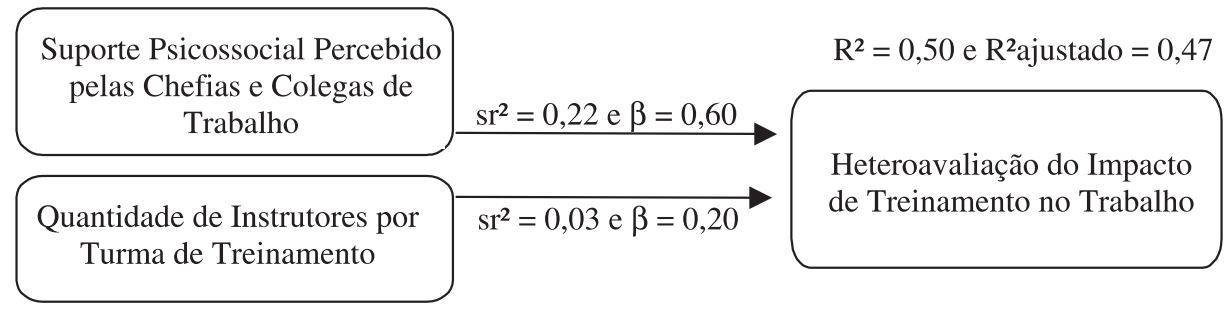

\section{Discussão e Comentários Finais}

Como foi observado, a variável que explicou a maior parte da variabilidade de auto-avaliação do impacto do treinamento no trabalho foi suporte psicossocial à transferência percebido pelos participantes, corroborando os achados de Roullier e Goldstein (1993), Abbad (1999), Brito (1999) e Sallorenzo (2000). Entre as características da clientela, auto-eficácia foi a única que explicou uma porção 
significativa das respostas de impacto, confirmando resultados descritos por Tannenbaum et al. (1991) e Colquit, LePine e Noe (2000).

Ao contrário de auto-eficácia para itens favoráveis, auto-eficácia para itens desfavoráveis não contribuiu significativamente na explicação de impacto do treinamento no trabalho. Talvez isso se deva, em parte, ao padrão de respostas dos participantes às escalas correspondentes às variáveis antecedentes. No caso de auto-eficácia para itens favoráveis, o padrão benevolente de respostas foi muito semelhante ao de impacto do treinamento. Em relação à escala de auto-eficácia para itens desfavoráveis, as respostas foram mais bem distribuídas nos cinco pontos de julgamento. Seus itens, por levarem a julgamentos desfavoráveis de auto-eficácia, podem ter tornado confusa a tarefa do avaliador, freqüentemente não acostumado a situações similares. Assim, caso os treinandos tivessem alto índice de auto-eficácia, os julgamentos deveriam variar entre os pontos mais baixos da escala. Vale ressaltar a necessidade de que novos estudos aperfeiçoem as escalas.

Apesar de auto-eficácia para itens favoráveis ter predito impacto do treinamento, observou-se, contrariamente aos resultados apontados na literatura, que a sua contribuição (2\%) para a explicação da variabilidade da variável critério é pequena e inferior à encontrada em outros estudos. É provável que este resultado se deva, em parte, ao fato de os itens conterem descrições de crenças muito genéricas que diferem das encontradas em outras pesquisas, nas quais auto-eficácia é definida para situações e contextos específicos. Surge então a necessidade de construir escalas de auto-eficácia específicas para a situação de transferência de treinamento, de modo que o participante possa avaliar suas capacidades de obter sucesso ao aplicar, no trabalho, as habilidades adquiridas em treinamento. Essa crença seria também de natureza disposicional, moldada a partir de experiências de sucessos e fracassos, porém de caráter mais específico que o encontrado na escala descrita neste estudo.

A natureza disposicional do conceito de auto-eficácia implica uma necessidade de acompanhamento de longo prazo das ações humanas que o representam, de modo a examinar de que maneira essa disposição se manifesta em diferentes situações ou contextos. Estudos de corte longitudinal ou de múltiplos cortes transversais são os delineamentos mais adequados para o estudo desse tipo de fenômeno. É sugerida a aplicação do questionário de auto-eficácia em mais de um ponto no tempo, antes do início do curso, no final do treinamento e após o treinamento, por exemplo. Com isso, poder-se-ia analisar a estabilidade da medida e, ao mesmo tempo, examinar cuidadosamente os efeitos do treinamento sobre os níveis de auto-eficácia, controladas as eventuais diferenças encontradas no pré-teste de auto-eficácia. 
Outra hipótese para a baixa contribuição de auto-eficácia na explicação de impacto é que, como sugerido por Colquit, LePine e Noe (2000), auto-eficácia estaria relacionada indiretamente a impacto do treinamento por mecanismos de motivação para aprender. No estudo realizado pelos autores, auto-eficácia prétreinamento esteve relacionada moderadamente com motivação para aprender (coeficiente de trajetória $=0,29)$ que, por sua vez, se relacionou também moderadamente com auto-eficácia pós-treinamento (coeficiente de trajetória $=0,22$ ). $\mathrm{O}$ relacionamento entre transferência do treinamento e auto-eficácia pós-treinamento foi de $r=0,27$. No presente estudo, não foram realizadas análises de relacionamentos indiretos entre variáveis; porém observou-se uma correlação bivariada de $r=0,37$ entre motivação e auto-eficácia (itens favoráveis), mais alta do que a correlação entre auto-eficácia e impacto $(r=0,19)$ e do que a correlação entre motivação e impacto $(r=0,17)$. Esses resultados sugerem que $o$ relacionamento entre auto-eficácia e motivação deve ser mais bem estudado a partir de modelos motivacionais de avaliação de treinamento, como o proposto pelos autores supracitados, em que variáveis como locus de controle e autoeficácia exercem influência indireta sobre resultados de treinamento.

Em relação às demais variáveis antecedentes, apesar de nenhuma delas ter contribuído na explicação de impacto do treinamento no trabalho, internalidade em locus de controle merece alguma atenção, principalmente devido aos resultados descritos por Abbad et al. (2001). Como sugerido por estes autores, autoeficácia e internalidade em locus de controle, quando submetidos conjuntamente a análises fatoriais, revelaram estrutura unifatorial, em que os itens de auto-eficácia possuíam cargas fatoriais mais elevadas do que os itens de locus de controle. Quando submetido a análises de regressão múltipla como variável antecedente distinta, locus de controle não explicou impacto de treinamento. Talvez isso se deva à redundância entre as variáveis antecedentes de auto-eficácia e internalidade. Nesses casos, uma das variáveis é excluída indevidamente da equação de regressão múltipla (Abbad e Torres, 2002).

Pesquisas posteriores deverão, além de examinar as distinções conceituais entre essas variáveis, submetê-las a análises fatoriais, na tentativa de confirmar a estrutura unifatorial sugerida e, nesse caso, transformá-la em variável preditora em modelos de regressão múltipla, em que impacto do treinamento no trabalho seja novamente a principal variável critério.

Outra possível explicação para a não contribuição de locus de controle na explicação de impacto do treinamento no trabalho é que, como sugerido por Colquit, LePine e Noe (2000), locus de controle está relacionado à transferência e à impacto do treinamento por meio de mediações por auto-eficácia pós-treinamento (coeficiente de trajetória $=0,49$ ) 
Quanto a suporte psicossocial à transferência, sua contribuição para predição de impacto do treinamento no trabalho já é bastante conhecida e ressaltada na literatura científica. Treinandos que acreditam dispor de ambiente que os apóia, têm seus desempenhos melhorados após a participação em treinamentos. Isso indica que quando os indivíduos, passados alguns meses após o curso, acreditam dispor de um ambiente apoiador, têm seus desempenhos melhorados.

Quando a variável critério era heteroavaliação de impacto do treinamento no trabalho, as variáveis antecedentes que contribuíram significativamente para a explicação de impacto foram, mais uma vez, suporte psicossocial, neste caso tal como percebido pelas chefias e colegas de trabalho, além da quantidade de instrutores por turma de treinamento, de modo que quanto maior o número de instrutores em um curso, maior o impacto do treinamento no trabalho. Isso pode ter ocorrido em função da eventual diversificação de estratégias de ensino que, provavelmente, aumentou as chances de o participante adquirir e generalizar novos conhecimentos para o trabalho. A presença de mais de um instrutor pode ter tornado o treinamento mais estimulante e motivador do que nos cursos ministrados por um só instrutor.

É importante observar que, no caso da heteroavaliação, auto-eficácia não contribuiu para a explicação da variabilidade de impacto do treinamento no trabalho, como ocorreu no modelo de auto-avaliação. É provável que o modo como os itens de auto-eficácia foram redigidos tenha inviabilizado o acesso dos treinandos a comportamentos ilustrativos de seus níveis de auto-eficácia. Estudos futuros devem construir indicadores comportamentais de auto-eficácia, que poderão ser aplicados a pessoas com alta e baixa auto-eficácia, de modo a identificar o que dizem e fazem indivíduos considerados auto-eficazes. Os itens assim descritos em termos de comportamentos manifestos poderão ser observados e julgados, além do próprio indivíduo, por outras pessoas.

Vale frisar que quando heteroavaliação de impacto foi utilizada como variável critério, somente suporte psicossocial percebido pelas chefias e colegas de trabalho e quantidade de instrutores por treinamento contribuíram significativamente para a sua explicação.

Apesar de a principal questão deste estudo estar relacionada à contribuição de variáveis individuais na predição de impacto de treinamento, os resultados mostraram que não são essas variáveis as principais preditoras de impacto. Quando medidas de avaliação auto-referente compuseram o modelo de regressão em conjunto com medidas referentes a aspectos externos e não relacionados à personalidade ou ao estilo pessoal, apenas as avaliações do ambiente organizacional explicaram significativamente o impacto do treinamento no trabalho. 
Ainda assim, os resultados desta pesquisa mostraram que a maior parte das variáveis incluídas no modelo de regressão múltipla é de natureza perceptual e que todas as variáveis explicativas de impacto são também de natureza perceptual. Talvez isso indique que a contribuição das variáveis antecedentes na explicação da variável critério se deva, em grande parte, à natureza das medidas aqui utilizadas e não à real importância dessas variáveis antecedentes na predição de impacto. Os resultados, nesse caso, estariam evidenciando padrões sistemáticos de respostas aos instrumentos, mais do que buscando referir-se aos fenômenos estudados.

Como ressalta Abbad (1999) sobre a desconfiança do papel da natureza das medidas na predição de impacto, os resultados obtidos por ela indicam que a aprendizagem, única medida não perceptual de desempenho (notas em testes de conhecimento), não esteve relacionada a impacto do treinamento no trabalho, apesar de a literatura sobre o assunto ressaltar geralmente sua contribuição importante na explicação de impacto. Mais estudos são necessários para reduzir os problemas e eliminar os vieses metodológicos dessa linha de pesquisa.

Os dados sobre a importância de suporte à transferência são, até agora, os mais robustos e objetivos. Mesmo em heteroavaliações de impacto, essa variável, relativa ao ambiente, é a mais forte preditora da variável critério em foco. Nesse caso, os respondentes parecem convergir em suas respostas, produzindo avaliações similares do ambiente no qual trabalham.

Por fim, é sugerido que pesquisas ou trabalhos futuros comparem resultados entre heteroavaliações, auto-avaliações e indicadores objetivos dessas variáveis. Ressalta-se também a importância da utilização de modelos mais sofisticados de análise de dados, como modelagem por equação estrutural, de modo a examinar com cuidado os fenômenos de moderação e mediação entre variáveis.

\section{Nota}

'Os instrumentos de Auto-eficácia e Locus de Controle foram desenvolvidos por um grupo de pesquisas, constituído por professores, alunos de pós-graduação e graduação do Departamento de Psicologia Social e do Trabalho da Universidade de Brasília, sob a coordenação da Profa. Dra. Gardênia Abbad. 


\section{ReferênCias Bibliográficas}

ABBAD, G.

Um modelo integrado de avaliação do impacto do treinamento no trabalho - IMPACT. Brasília, 1999. Tese (Doutorado em Psicologia) - Instituto de Psicologia, Universidade de Brasília.

ABBAD, G.;

BORGES-ANDRADE, J. E.;

SALLORENZO, L. H.

Self-assessment of training impact at work - a conceptual analyses. manuscrito não publicado.

ABBAD, G. et al.

Confusão conceitual entre medidas de auto-eficácia e locus de controle em uma amostra de treinandos de uma empresa do setor de telecomunicações. In: REUNIÃO ANUAL DE PSICOLOGIA, 31., 2001, Rio de Janeiro. Anais... Rio de Janeiro: Sociedade Brasileira de Psicologia, 2001. p. 246.

ABBAD, G.;

TORRES, C. V.

Regressão múltipla em estudos de psicologia organizacional: problemas e algumas soluções. Estudos de Psicologia. No prelo.

BASTOS, A. V. B.

Validação da escala locus de controle no trabalho. PSICO, v. 22, n. 2, p. 133-154, 1991.
BORGES-ANDRADE, J. E.

Avaliação somativa de sistemas instrucionais: integração de três propostas. Tecnologia Educacional, ano XI, v. 11, n. 46, p. 2939, 1982.

Treinamento de pessoal: em busca de conhecimento e tecnologia relevantes para as organizações brasileiras. In: TAMAYO, A.; BORGES-ANDRADE, J. E.; CODO, W. (Eds). Trabalho, organizações e cultura. São Paulo: Cooperativa de Autores Associados, 1997. p. 129-149.

BORGES-ANDRADE, J. E.; ABBAD, G.

Treinamento e desenvolvimento: reflexões sobre suas pesquisas científicas. Revista de Administração, v. 31 , n. 2, p. 112-125, 1996.

BRITTO, M. J. P.

Avaliação de impacto de treinamentos na área de reabilitação: preditores individuais e situacionais. Brasília, 1999. Dissertação (Mestrado) - Universidade de Brasília. 
COLQUIT, J. A.;

LEPINE, J. A.;

NOE, R. A.

Toward an integrative theory of training motivation: a meta-analytic path analysis of 20 years of research. Journal of Applied Psychology, v. 85, n. 5 , p. $678-707,2000$.

\section{GOLDSTEIN. I. L.;}

GILLIAM, P.

Training system issues in the year 2000. American Psychologist, v. 45, p. 134-143, 1990.

HAMBLIN, A. C. Avaliação e controle de treinamento. São Paulo: McGrawHill do Brasil, 1978.

\section{LATHAM, G. P.}

Human resource training and development. Annual Review of Psychology, v. 39, p. 545-582, 1988.

\section{LEITÃO, J. S. S.}

Relações entre clima organizacional e transferência de treinamento. Brasília, 1994. Dissertação (Mestrado) - Universidade de Brasília.

\section{MENESES, P. P. M.}

Auto-eficácia, locus de controle, suporte à transferência e impacto do treinamento no trabalho. Brasília, 2002. Dissertação (Mestrado) - Universidade de Brasília.

PAULA, S. M. A.

Variáveis preditoras de impacto do treinamento no trabalho: análise da percepção dos treinandos de duas organizações. Brasília, 1992. Dissertação (Mestrado) - Universidade de Brasília.

ROULLIER, J. Z.;

GOLDSTEIN, I. L.

The relationship between organizational transfer climate and positive transfer of training. Human Resource Development Quaterly, v. 4, n. 4, p. 377-390, 1993.

SALAS, E.;

CANNON-BOWERS, A.

The science of training: a decade of progress. Annual Review of Psychology, v. 52, p. 471-499, 2001.

SALLORENZO, L. H.

Avaliação de impacto de treinamento no trabalho: analisando e comparando modelos de predição. Brasília, 2000. Dissertação (Mestrado) - Universidade de Brasília.

TANNENBAUM, S, I. et al.

Meeting trainee's expectations: the influence of training fulfillment on the development of commitment, self-efficacy, and motivation. Journal of Applied Psychology, v. 76, n. 6, p. 759-769, 1991.

TANNENBAUM, S. I.;

YUKL, G.

Training and development in work organizations. Annual Review of Psychology, v. 43, p. 399-441, 1992. 